\title{
Histamine-2 receptor antagonist famotidine modulates cardiac stem cell characteristics in hypertensive heart disease
}

\author{
Sherin Saheera ${ }^{1}$, Ajay G Potnuri ${ }^{1}$, Renuka Nair ${ }^{\text {Corresp. } 1}$ \\ ${ }^{1}$ Division of Cellular and Molecular Cardiology, Sree Chitra Tirunal Institute for Medical Sciences and Technology, Thiruvananthapuram, Kerala, India \\ Corresponding Author: Renuka Nair \\ Email address: renukanairr52@gmail.com
}

Background: Cardiac stem cells (CSCs) play a vital role in cardiac homeostasis. Decrease in efficiency of cardiac stem cells is speculated in various cardiac abnormalities. The maintenance of a healthy stem cell population is essential for prevention of adverse cardiac remodeling leading to cardiac failure. Famotidine, a histamine-2 receptorantagonist is currently used to treat ulcers of the stomach and intestines. Repurposing the use of the drug, reduction of cardiac hypertrophy and improvement in cardiac function of Spontaneously hypertensive rat (SHR) was reported by our group. Given that stem cells are affected in cardiac pathologies, the effect of histamine-2 receptor antagonism on CSC characteristics was investigated. Methods: To examine whether famotidine has a positive effect on CSCs, Spontaneously hypertensive rat (SHR) treated with the drug were sacrificed; and CSCS isolated from atrial appendages was evaluated. Six-month-old male SHR were treated with famotidine (30 mg/kg/day) for 2 months. The effect of famotidine treatment on migration, proliferation and survival of CSCs was compared with untreated SHR and normotensive Wistar rat. Results: Functional efficiency of CSCs from SHR was compromised relative to that in Wistar rat. Famotidine increased the migration and proliferation potential, along with retention of stemness of CSCs in treated SHR. Cellular senescence and oxidative stress were also reduced. The expression of H2R was unaffected by the treatment. Discussion: As anticipated, CSCs from SHR were functionally impaired. Stem cell attributes of famotidine treated SHR wascomparable to that of Wistar rat. Therefore, in addition to being cardioprotective, the histamine 2 receptor antagonist modulated cardiac stem cells characteristics. Restoration of stem cell efficiency by famotidine is possibly mediated by reduction of oxidative stress as the expression of $\mathrm{H} 2 \mathrm{R}$ was unaffected by the treatment. Maintenance of healthy stem cell population is suggested as a possible mechanism underlying the cardioprotective effect of famotidine. 
1

2

3

4 Division of Cellular and Molecular Cardiology, Sree Chitra Tirunal Institute for Medical

5 Sciences and Technology, Trivandrum, Thiruvananthapuram - 695011, Kerala, India.

6 *Corresponding author:

7 Dr. Renuka R Nair

8 Senior Scientist G,

9 Division of Cellular and Molecular Cardiology,

10 Sree Chitra Tirunal Institute for Medical Sciences and Technology,

11 Trivandrum, Kerala, India.

12 Tel: +914712524505 .

13 Email: renukanairr52@gmail.com

\section{Abstract}

15 Background: Cardiac stem cells (CSCs) play a vital role in cardiac homeostasis. Decrease in 16 efficiency of cardiac stem cells is speculated in various cardiac abnormalities. The maintenance

17 of a healthy stem cell population is essential for prevention of adverse cardiac remodeling leading

18 to cardiac failure. Famotidine, a histamine-2 receptor antagonist is currently used to treat ulcers

19 of the stomach and intestines. Repurposing the use of the drug, reduction of cardiac hypertrophy 
20 and improvement in cardiac function of Spontaneously hypertensive rat (SHR) was reported by

21 our group. Based on the premise that stem cells are affected in the pathological heart, the effect

22 of histamine-2 receptor antagonism on CSC characteristics was investigated.

23 Methods: To examine whether famotidine has a positive effect on CSCs, Spontaneously

24 hypertensive rat (SHR) treated with the drug were sacrificed; and CSCs isolated from atrial

25 appendages was evaluated. Six-month-old male SHR were treated with famotidine (30

$26 \mathrm{mg} / \mathrm{kg} /$ day) for 2 months. The effect of famotidine treatment on migration, proliferation and

27 survival of CSCs was compared with untreated SHR and normotensive Wistar rat.

28 Results: Functional efficiency of CSCs from SHR was compromised relative to that in Wistar rat.

29 Famotidine increased the migration and proliferation potential, along with retention of stemness

30 of CSCs in treated SHR. Cellular senescence and oxidative stress were also reduced.

31 The expression of H2R was unaffected by the treatment.

32 Discussion: As anticipated, CSCs from SHR were functionally impaired. Stem cell attributes of

33 famotidine treated SHR was comparable to that of Wistar. In addition to being cardioprotective,

34 the histamine 2 receptor antagonist modulated cardiac stem cell characteristics. Restoration of

35 stem cell efficiency by famotidine is possibly mediated by reduction of oxidative stress as the

36 expression of $\mathrm{H} 2 \mathrm{R}$ was unaffected by the treatment. Maintenance of healthy stem cell population

37 is suggested as a possible mechanism underlying the cardioprotective effect of famotidine.

\section{Introduction}

39 The discovery of cardiac stem cells challenged the notion of heart being a post-mitotic organ

40 (Beltrami et al., 2003). Cardiomyocyte turnover ranges from 0.5-1\% annually (Bergmann et al.,

41 2009), implicating the involvement of cardiac stem cells in the maintenance of cardiac

42 homeostasis. Protecting resident cardiac stem cells as a prelude to prevention of cardiac failure

43 has not received much attention. The adverse microenvironment and the cycling of stem cells for 
44 replenishment of lost myocytes in the pathological heart can lead to stem cell aging. In the event

45 of myocardial injury, cardiac stem cells mediate tissue repair and regeneration. The positive effect

46 of stem cell transplantation for myocardial regeneration highlights the role of stem cells in tissue

47 repair (Smits et al., 2005). Impaired efficiency of human cardiac stem cells has been documented

48 in pathological conditions (Cesselli et al., 2011). Studies on the influence of cardioprotective

49 drugs on cardiac stem cells are limited. Comparison of the effects of an angiotensin receptor

50 blocker losartan and a beta-blocker metoprolol on left ventricular (LV) remodeling, in rat surgical

51 model showed that along with improvement in LV function, the number of c-kit ${ }^{+}$cells as well as

52 expression of Ki-67 was increased by metoprolol but not losartan (Serpi et al., 2009). Therefore,

53 selective cardio protective drugs are envisaged to modulate stem cell characteristics, to maintain

54 a healthy stem cell pool.

55 Histamine, secreted by mast cells in the heart, has been implicated in cardiac diseases and the 56 development of heart failure (Francis \& Tang, 2006). Histamine receptors are present widely in

57 the heart (Felix et al., 1988). We have reported that the $\mathrm{H} 2$ receptor antagonist famotidine 58 promotes reverse cardiac remodeling in Spontaneously hypertensive rat (SHR) (Potnuri et al., 59 2016). Though impairment of CSCs is implicated in cardiac pathologies, modulation of stem cell 60 characteristics by famotidine has not been reported. Hence, this study was carried out based on 61 the assumption that famotidine will restore the cardiac stem cell efficiency, that is compromised 62 in SHR.

\section{Materials and methods}

\section{$64 \quad 2.1$ Experimental design}

65 Six-month old SHR was used as the experimental model. Twelve male SHR were randomly 66 assigned into two groups of six rats each. One group of untreated SHR served as hypertensive 67 control and the other group received a daily oral dose of $30 \mathrm{mg} \cdot \mathrm{kg}^{-1}$.day ${ }^{-1}$ of famotidine for 2 68 months. The response to treatment was evaluated by comparison with stem cell characteristics of 
69 untreated SHR and normotensive Wistar rat (WST). The animals were housed at $22^{\circ} \mathrm{C}$,

70 maintained on a $12 \mathrm{~h}$ light-dark cycle, fed with regular Rat Chow and had free access to drinking

71 water. All animal procedures were approved by the Sree Chitra Tirunal Institute for Medical

72 Sciences and Technology, Institutional Animal Ethics Committee, according to the Committee for

73 the Purpose of Control and Supervision of Experiments on Animals (CPCSEA) Guidelines.

74 (Approval Reference No. B 2422012 XXI). The stem cell research was approved by The Sree

75 Tirunal Institute for Medical Sciences and Technology, Institutional Committee for stem cell

76 research- (Approval No. SCT/IC-SCRT/01/Mar-2012).

\section{2.2. Isolation, characterisation and expansion of CSCs}

78 Atrial explants were established in $2 \%$ gelatin coated dishes supplemented with IMDM and $10 \%$

79 FBS. c-kit ${ }^{+}$CSCs were isolated immunomagnetically using Easy Sep Magnet and Easy Sep

80 FITC positive selection kit (Stem Cell Technologies). The CSCs were expanded in culture

81 medium containing IMDM along with $10 \% \mathrm{FBS}, 10 \mathrm{ng} / \mathrm{ml}$ basic Fibroblast growth factor, $500 \mu 1$

82 Insulin- selenium-transferrin along with the antibiotics penicillin and gentamycin. The cells in

83 passage 3 were assessed for their purity based on expression of cell surface markers $-\mathrm{c}-\mathrm{kit}, \mathrm{CD}$

84 45, CD34 and CD 31using flow cytometry and immunocytochemistry. The cells from the third

85 passage were used for further studies.

86 2.3. Colony Forming Unit Assay

87 Self renewing ability is a characteristic of stem cells. CSCs were plated at a cell density of 500

88 cells per $60 \mathrm{~mm}$ culture plate. After 14 days, the cells were washed with PBS and stained with $3 \%$

89 crystal violet in methanol for 30 minutes at room temperature. The colonies larger than $2 \mathrm{~mm}$

90 were counted.

91 2.4. Proliferation Capacity 
92 Cells were plated at a density of 10000 cells per plate (Zhang et al., 2013). The cell number was

93 obtained every $48 \mathrm{hrs}$ till the $10^{\text {th }}$ day. Growth kinetics was obtained by plotting the cell count.

94 Growth rate (GR) and Population doubling time (PDT) were calculated using standard

95 mathematical formulae. $\mathrm{GR}=\ln \left(\mathrm{N}_{\mathrm{t}} / \mathrm{N}_{0}\right) / \mathrm{T}$, where $\mathrm{T}$ is the incubation time, $\mathrm{N}_{0}$ is the cell number at

96 the beginning of the incubation time and $\mathrm{N}_{\mathrm{t}}$ is the cell number at the end of the incubation time.

97 Population doubling time was calculated using the formula, $\mathrm{PDT}=\ln (2) / \mathrm{GR}$.

98 2.5. Migration Ability

99 The migration potential was assessed by trans-well migration assay using serum as chemo-

100 attractant. A total of $1 * 10^{4}$ cells were seeded onto the upper chamber of the trans-well ( BD 101 Falcon, pore size $-8 \mu \mathrm{m}$ ) in serum free medium. IMDM containing $10 \%$ serum was added in the 102 lower chamber, where serum acted as the chemoattractant. Following incubation for 18 hrs, the 103 cells on the upper surface of the membrane were removed and those that migrated to the lower 104 surface were fixed with 4\% paraformaldehyde, stained with crystal violet and counted.

\section{2.6. Intracellular Reactive oxygen species levels}

106 The intracellular reactive oxygen species (ROS) levels in CSCs were determined from $\mathrm{H}_{2}$ DCFDA 107 fluorescence intensity (LeBel, Ischiropoulos \& Bondy, 1992). CSCs were incubated for 10 min 108 with $10 \mu \mathrm{M}$ of $\mathrm{DCFH}_{2} \mathrm{DA}$ in DMSO. The fluorescence intensity of DCF was measured using 109 Microplate reader. Fluorescence values were monitored by excitation at $498 \mathrm{~nm}$ and emission at $110530 \mathrm{~nm}$.

\section{2.7. Senescent CSCs}

112 The proportion of senescent cells was assessed based on the cytochemical analysis of senescence 113 associated $\beta$-galactosidase staining using commercially available kits (Abcam). The cells that 114 stained positive for $\beta$-galactosidase were counted with the help of light microscope. A minimum 
115 of 5 random fields from each dish with a minimum of 500 cells were counted and percentage of

116 senescent cells was calculated.

\section{2.8. Western Blot analysis for expression of Histamine 2 receptor in CSCs}

118 Twentyfive micrograms of protein from each sample was electrophoresed on $10 \%$ 119 polyacrylamide SDS gel along with the histamine-2 receptor peptide. After transferring proteins 120 to nitrocellulose membranes, they were incubated with the primary antibodies followed by 121 appropriate secondary antibodies. Protein bands were visualized by chemi-luminescence and

122 images were quantified using image $\mathbf{J}$ software. The expression of target protein was normalised 123 to their respective beta actins.

\section{Statistical analysis}

125 Values are presented as mean \pm SD. One way ANOVA was carried out and if there was significant 126 variation between samples, Student t-test was used for evaluation of differences between 127 samples. Results were considered statistically significant for $\mathrm{p}$ values less than 0.05 .

\section{Results}

\section{3.1. Isolation, Culture and characterization of CSCs}

130 Following famotidine treatment for 60 days, animals were sacrificed, hearts were dissected under 131 aseptic conditions and atrial tissue was cultured as explants. Small, phase bright cells migrated 132 from the explants within 14 days. The cells were trypsinised and subjected to immunomagnetic 133 isolation to sort out the c-kit ${ }^{+}$and lineage negative CSCs. The purity of the cultured CSCs was 134 confirmed at passage 3 by flow cytometry and immunocytochemistry. The analysis revealed that $13592 \pm 3 \%$ cells were positive for c-kit and negative for hematopoietic, endothelial and mast cell 136 markers CD45, CD31 and CD 34 respectively (Fig.1). The cultured CSCs in the third passage 
137 were used for further experiments. The cells so isolated were able to form colonies when seeded 138 at the rate of 0.5 cell/well in 96 well-plate further confirming their stem cell characteristics.

\section{3.2. Effect of famotidine on self renewing ability of CSCs}

140 Colony formation is characteristic of stem cells. The number of colonies formed from CSCs of

141 SHR was 68\% lower than that from WST. Famotidine treatment improved the self renewing 142 capacity of CSCs and the values were comparable with that of WST (Fig2).

\section{3.3. Effect of famotidine on proliferation capacity of CSCs}

144 Proliferation capacity as assessed by cell number, growth rate and PDT was significantly lower in 145 SHR, reiterating the compromised efficiency of CSCs in hypertensive heart disease. The 146 proliferation capacity of CSCs from SHR improved significantly following treatment with 147 famotidine (Fig 3).

\section{3.4. Effect of famotidine on migration ability of CSCs}

149 The migration rate of CSCs was 38\% lower in SHR compared to WST, when assessed by trans150 well assay. The lower infiltration towards the chemo-attractant serum reiterates their 151 compromised efficacy. However, following treatment with famotidine, the migration potential 152 improved by $15 \%$ indicating the beneficial effect of the drug (Fig 4B-E).

\section{3.5. Effect of famotidine on intracellular ROS levels of CSCs}

154 Extracellular ROS, the major contributor towards hypertophic remodelling in SHR, influences the 155 functional efficiency of CSCs by interacting with their microenvironment/niche. The intracellular 156 ROS levels of CSCs as assessed by $\mathrm{H}_{2}$ DCFDA fluorescence intensity, was significantly high in 157 SHR and regressed on treatment with famotidine indicating the antioxidant effect of the drug (Fig 158 4A).

159 3.6. Effect of famotidine on senescence of CSCs 
160 Efficiency of CSCs is remarkably compromised in the presence of senescent cells. The proportion

161 of senescent cells as assessed by SA- $\beta$-galactosidase staining was high in SHR compared to age-

162 matched WST, but regressed significantly on treatment with famotidine (Fig 5A-D).

\section{$163 \quad 3.7 . \quad$ Expression of Histamine 2 receptor in CSCs}

164 Western blot analysis of the protein samples revealed the presence of histamine-2- receptor in 165 CSCs. Upon normalization with $\beta$-actin, the expression levels of H2R was similar between age 166 matched WST and SHR (Fig. 5 E,F) The treatment did not alter the expression of the receptor.

167 4. Discussion

168 Hypertensive heart disease progresses through cardiac hypertrophy paving the way to heart 169 failure, making it a major shareholder of all the mortalities worldwide. Cardiac hypertrophy 170 involves the enlargement of cardiomyocytes along with increased fibrosis and decreased capillary 171 density. Though cardiac remodelling starts as an adaptive response, in course of time it becomes 172 maladaptive and the heart fails to counterbalance the pressure overload. Many physiological, 173 cellular and molecular mechanisms play an interconnecting role in inducing remodelling. Of the 174 several mechanisms predicted, oxidative stress is considered to be the critical determinant; being 175 both the cause and consequence of pathological changes in cardiac hypertrophy. Studies from our 176 laboratory has shown that oxidative stress precedes the hypertrophic remodeling in SHR and is 177 evident as early as 1 month of age ( Purushothaman et al., 2011).

178 The cardiac stem cells were discovered in 2003 and ever since, studies have been extensively 179 carried out on c-kit+ cells. The endogenous stem cells in the heart play a critical role in 180 maintaining cardiac homeostasis and regeneration, (Bergmann et al., 2009) highlighting the 181 significance of this minor population of cells. Studies have implicated the prominent role of 182 cardiac stem cells in mediating tissue repair (Nadal-Ginard, Ellison \& Torella, 2014). Very little 183 information is available on the impact of pathological conditions on resident CSCs and the 184 influence of cardioprotective drugs on modulating stem cell characteristics. The adverse 185 microenvironment prevailing in the pathological heart can affect the efficient functioning of 
186 resident cardiac stem cells. Therefore, a conducive microenvironment is expected to protect the

187 heart by modulation of stem cell characteristics. A study carried out in our laboratory showed 188 that the anti-ulcer drug famotidine, a histamine-2 receptor antagonist, regressed hypertrophy

189 with evident morphological and molecular changes (Potnuri et al., 2016). Other studies also 190 support the role of histamine-2 receptor antagonism in prevention of heart failure (Francis \& 191 Tang, 2006). Finding improvement in cardiac structure and function in SHR, we chose to 192 examine whether famotidine modulated the CSCs.

193 Heart was procured during the sacrifice of animals treated with famotidine for 2 months. Atria

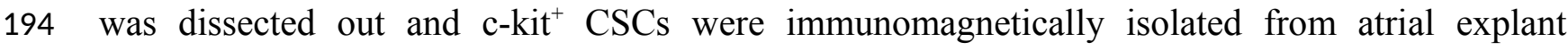
195 cultures. The CSCs were then expanded in culture. Assessment of the purity and stemness of the 196 CSCs at passage 3 confirmed the cardiac origin of these cells (Fig. 1) as they were positive for c197 kit and negative for haematopoietic (CD 45), endothelial (CD 31) and mast cell (CD 34) markers. 198 (Okayama \& Kawakami, 2006; Dahlin \& Hallgren, 2015) Since the yield of CSCs from the 199 primary isolation was low, the cells were expanded in culture to obtain cells in sufficient numbers 200 for evaluation of the variables. Studies have shown that long term culture, upto passage 40 201 maintains the c-kit nature/stemness of CSCs (Miyamoto et al., 2010). The cells in the third 202 passage were assessed for stem cell characteristics.

203 The ability to form colonies is a characteristic feature of stemness. CSCs from SHR, formed 204 lower number of colonies compared to their age matched WST. Nevertheless, colony formation 205 ability improved significantly in response to the treatment (Fig 2) indicating restorationn of 206 stemness. Maintaining stem cells in their quiescent state within the niche will help to preserve the 207 growth reserve of the heart. CSCs from SHR had compromised proliferation capacity as evident 208 from the decreased growth kinetics and increased PDT. Increase in growth kinetics (Fig 3 A-C) 209 upon treatment signifies the revival of proliferation efficiency and improved efficacy of the 210 CSCs. Decreased proliferation and colony formation implies that CSCs from SHR in the stable 211 phase of hypertrophy are affected by maladaptive remodeling of the heart. Similar observations 
212 have been found when Adipose derived stem cells from Twitcher mice were compared with

213 normal mice. The Adipose derived stem cells from diseased mice exhibited less self-replicating

214 and proliferative capacity. (Zhang et al., 2013).

215 Homing in of stem cells to the site of injury is an essential component for tissue repair. The

216 functional efficiency of stem cells is determined by its ability to effectively invade and repair the

217 damaged tissue (GUO et al., 2014). The deficient migratory capacity of CSCs with hypertensive

218 heart disease implies functional impairment of CSCs for repair and regeneration upon demand.

219 The migration potential of CSCs in SHR was restored by famotidine, (Fig 4B-E) denoting

220 functional recovery. The improved invasive capacity is expected to aid the CSCs to effectively

221 home in to the site of injury and replace the damaged myocytes; that occur at a relatively higher

222 frequency in hypertrophic remodeling.

223 Stem cells, like somatic cells undergo aging process and express senescent markers (Chambers et

224 al., 2007; Akunuru \& Geiger, 2016). The presence of senescent cells in the niche can release

225 paracrine signals that adversely affect the neighboring healthy cells (Schellenberg et al., 2011).

226 The proportion of senescent cells was significantly higher in SHR compared with the age

227 matched WST (Fig 5A-D). Famotidine mediated decrease in senescence helps in preserving the

228 stem cell pool.

229 The positive response of CSCs to famotidine can be mediated either by modulation of the

230 microenvironment or the blockade of $\mathrm{H} 2 \mathrm{R}$, since famotidine is a $\mathrm{H} 2 \mathrm{R}$ antagonist. H2R receptor

231 is known to be present in stem cells and progenitor cells (Yamada et al., 2013; Liu et al., 2017),

232 but the presence of histamine-2-receptors in CSCs has not been reported. This is the first study to

233 report the presence of $\mathrm{H} 2 \mathrm{R}$ in CSCs. Though the CSCs expressed H2R, the levels of receptor was

234 not significantly different between SHR, WST and the treated group (Fig. 5 E,F) implicating the

235 role of other mechanisms for the improvement in the functionality of CSCs. This is also

236 supported by the fact that stem cells residing within the niches are resistant to drugs due to the

237 specific miroenvironment and hypoxic stability (Vinogradov \& Wei, 2012; Alonso, Jones \& 
238 Ghiaur, 2017). This can account for the lack of change in expression of H2R in SHR treated with

239 famoidine (Fig. 5. E,F).

240 The proposed mechanism of action of famotidine is the decrease in oxidative stress of the

241 microenvironment. Famotidine treatment decreased myocardial oxidative stress and improved

242 cardiac function, along with decrease of left ventricular wall thickness (Potnuri et al., 2016).

243 Oxidative stress is the key regulator of multiple pathways involved in hypertrophy, and is

244 detected at an early stage in SHR which prevails throughout the pathological remodelling

245 (Purushothaman et al., 2011). An adverse microenvironment can contribute to the aging of stem

246 cells. Enhanced oxidative stress in SHR can be the major determinant for the inefficient

247 functioning of stem cells. Though no reports on the intracellular ROS of CSCs is available,

248 oxidative stress has been reported to affect the endothelial progenitor cells (Case, Ingram \&

249 Haneline, 2008). The increased ROS levels in CSCs from SHR (Fig 4A) suggest that 250 intracellular oxidative stress can affect the overall efficiency of stem cells. The reduction of ROS

251 upon treatment further supports the notion that the improved efficacy of CSCs is due to decreased 252 oxidative stress both at the tissue and cellular levels. Hence, modulation of stem cell attributes 253 by famotidine is possibly mediated by reduction of oxidative stress.

\section{Conclusion}

255 The fate of an organ is determined by the reserve of functionally efficient stem cells. In 256 hypertensive heart disease, maintenance of a healthy stem cell population is expected to prevent 257 progressive cardiac remodeling. Famotidine mediated restoration of stem cell attributes therefore 258 gains significance. The improvement in stem cell efficiency is possibly mediated by reduction of 259 oxidative stress since the H2R expression in CSCs of SHR was comparable to that of Wistar and 260 was unaltered by treatment with famotidine. The modulation of stem cell efficiency by $\mathrm{H} 2$ 261 receptor antagonism lends scope for further investigations enabling therapeutic application for 
262 prevention of progressive cardiac remodeling. Retrospective and prospective studies on the

263 response to famotidine treatment has to be carried out for further validation of our findings.

\section{References}

265 Akunuru S., Geiger H. 2016. Aging, Clonality, and Rejuvenation of Hematopoietic Stem Cells. Trends in Molecular Medicine 22:701-712. DOI: 10.1016/j.molmed.2016.06.003.

Alonso S., Jones RJ., Ghiaur G. 2017. Retinoic acid, CYP26, and drug resistance in the stem cell niche. Experimental Hematology. DOI: 10.1016/j.exphem.2017.07.004.

Dahlin JS., Hallgren J. 2015. Mast cell progenitors: Origin, development and migration to tissues.

Chambers SM., Shaw CA., Gatza C., Fisk CJ., Donehower LA., Goodell MA. 2007. Aging Hematopoietic Stem Cells Decline in Function and Exhibit Epigenetic Dysregulation. PLOS Biology 5:e201. Molecular Immunology 63:9-17. DOI: 10.1016/j.molimm.2014.01.018.

Felix SB., Baumann G., Helmus S., Sattelberger U. 1988. The role of histamine in cardiac anaphylaxis; characterization of histaminergic H1- and H2-receptor effects. Basic Research in Cardiology $83: 531-539$. 
GUO J., JIE W., SHEN Z., LI M., LAN Y., KONG Y., GUO S., LI T., ZHENG S. 2014. SCF increases cardiac stem cell migration through PI3K/AKT and MMP-2/-9 signaling. International Journal of Molecular Medicine 34:112-118. DOI: 10.3892/ijmm.2014.1773.

LeBel CP., Ischiropoulos H., Bondy SC. 1992. Evaluation of the probe 2',7'-dichlorofluorescin as an indicator of reactive oxygen species formation and oxidative stress. Chemical Research in Toxicology 5:227-231. DOI: 10.1021/tx00026a012.

Liu X., Kumagai G., Wada K., Tanaka T., Fujita T., Sasaki A., Furukawa K-I., Ishibashi Y. 2017. Suppression of osteogenic differentiation in mesenchymal stem cells from patients with ossification of the posterior longitudinal ligament by a histamine-2-receptor antagonist. European Journal of Pharmacology 810:156-162. DOI: 10.1016/j.ejphar.2017.07.013.

Miyamoto S., Kawaguchi N., Ellison GM., Matsuoka R., Shin’oka T., Kurosawa H. 2010. Characterization of long-term cultured c-kit+ cardiac stem cells derived from adult rat hearts. Stem Cells and Development 19:105-116. DOI: 10.1089/scd.2009.0041.

Nadal-Ginard B., Ellison GM., Torella D. 2014. The cardiac stem cell compartment is indispensable for myocardial cell homeostasis, repair and regeneration in the adult. Stem Cell Research 13:615-630. DOI: 10.1016/j.scr.2014.04.008.

Okayama Y., Kawakami T. 2006. Development, Migration, and Survival of Mast Cells. Immunologic research 34:97-115.

Potnuri AG., Allakonda L., Appavoo A., Saheera S., Nair RR. 2016. Targeting histamine-2 receptor for prevention of cardiac remodelling in chronic pressure overload. International Journal of Cardiology 202:831-833. DOI: 10.1016/j.ijcard.2015.10.040.

Purushothaman S., Renuka Nair R., Harikrishnan VS., Fernandez AC. 2011. Temporal relation of cardiac hypertrophy, oxidative stress, and fatty acid metabolism in spontaneously hypertensive rat. Molecular and Cellular Biochemistry 351:59-64. DOI: 10.1007/s11010-011-0711-y.

Schellenberg A., Lin Q., Schüler H., Koch CM., Joussen S., Denecke B., Walenda G., Pallua N., Suschek CV., Zenke M., Wagner W. 2011. Replicative senescence of mesenchymal stem cells causes DNA- 
methylation changes which correlate with repressive histone marks. Aging (Albany NY) 3:873888.

317 Serpi R., Tolonen A-M., Tenhunen O., Pieviläinen O., Kubin A-M., Vaskivuo T., Soini Y., Kerkelä R., Leskinen H., Ruskoaho H. 2009. Divergent effects of losartan and metoprolol on cardiac remodeling, c-kit+ cells, proliferation and apoptosis in the left ventricle after myocardial infarction. Clinical and Translational Science 2:422-430. DOI: 10.1111/j.17528062.2009.00163.x.

Smits AM., van Vliet P., Hassink RJ., Goumans M-J., Doevendans PA. 2005. The role of stem cells in cardiac regeneration. Journal of Cellular and Molecular Medicine 9:25-36.

Vinogradov S., Wei X. 2012. Cancer stem cells and drug resistance: the potential of nanomedicine. Nanomedicine (London, England) 7:597-615. DOI: 10.2217/nnm.12.22. disease. BMC cell biology 14:20. DOI: 10.1186/1471-2121-14-20.

\section{Figures}

A

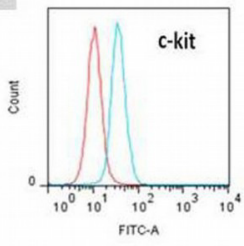

B

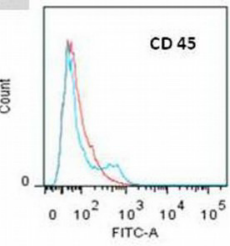

C

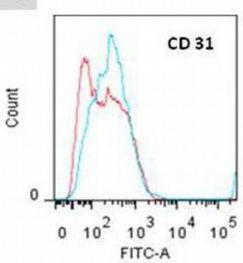

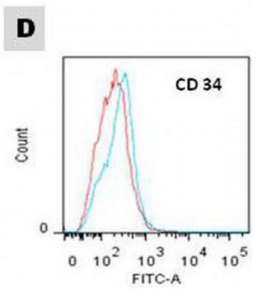

$\underline{\text { Percentage distribution of the surface markers }}$ 
333 Figure 1: Characterization of the cultured CSCs at passage 3.

334 (A-D) Representative FACS images and (E) the percentage distribution of specific markers.

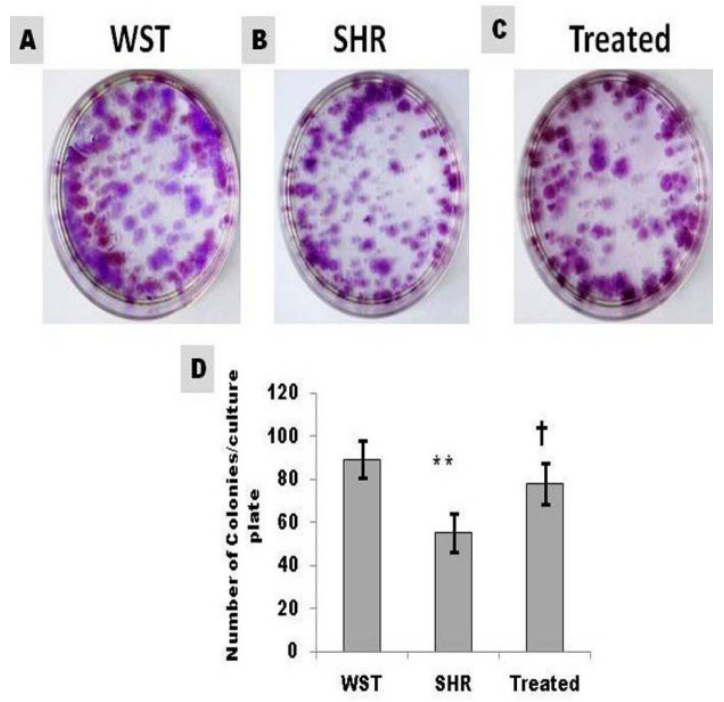

335 Figure 2: Effect of famotidine on self-renewing ability of atrial CSCs

336 (A-C) Representative images and (D) Graphical representation of Colony forming units (CFU) 337 shown as number of colonies/ culture plate $(60 \mathrm{~mm}$ diameter). Data presented as mean $\pm \mathrm{SD}$. 338 Variation was analyzed by ANOVA followed by Student t-test. (** $\mathrm{p}<0.01$ SHR Vs WST; $339 \uparrow \mathrm{p}<0.05$ SHR Vs Famotidine) ANOVA $\mathrm{p}<0.01$ ( $\mathrm{n}=6 /$ group).
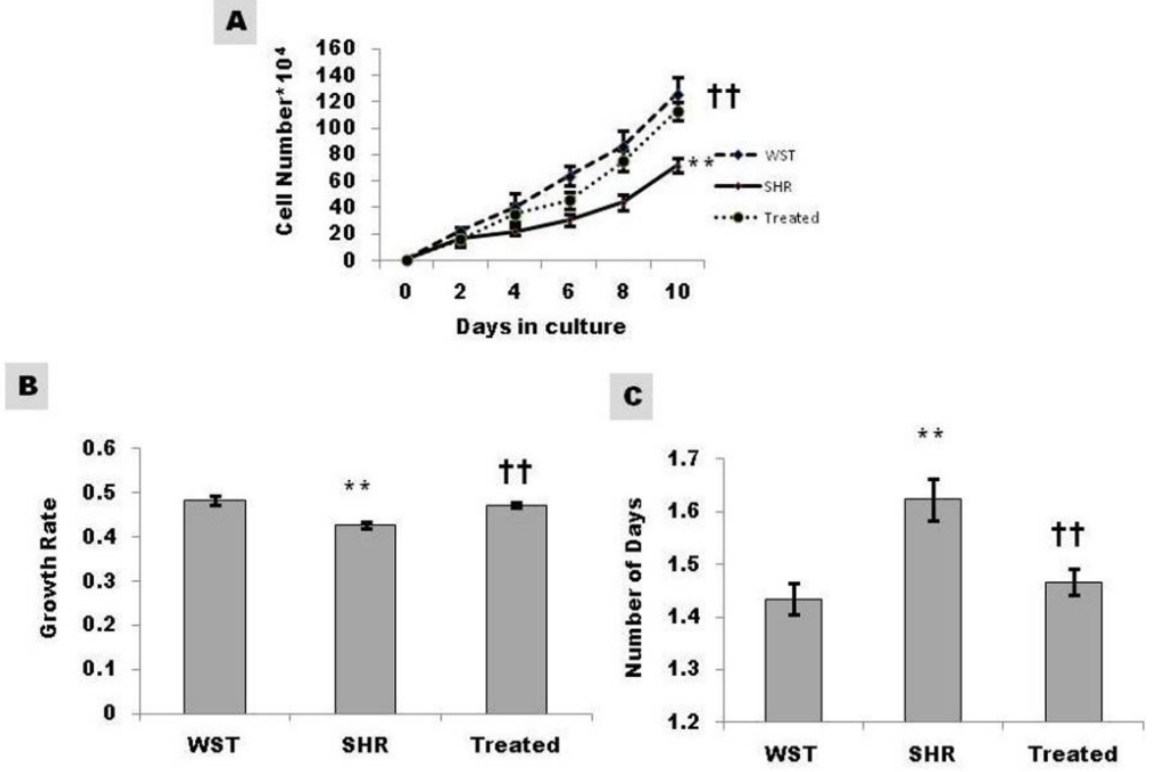

Figure 3: Effect of famotidine on growth kinetics, growth rate and population doubling time of atrial CSCs

A. Growth kinetics of CSCs represented as cell number* $10^{4}$

B. Growth rate calculated as $\log N$ 
345 followed by Student t-test. ( ${ }^{* *} \mathrm{p}<0.01$ SHR Vs WST; $\dagger \dagger \mathrm{p}<0.01 \& \dagger \mathrm{p}<0.05$ SHR Vs 346 Famotidine) ANOVA $\mathrm{p}<0.01$ ( $\mathrm{n}=6$ /group)
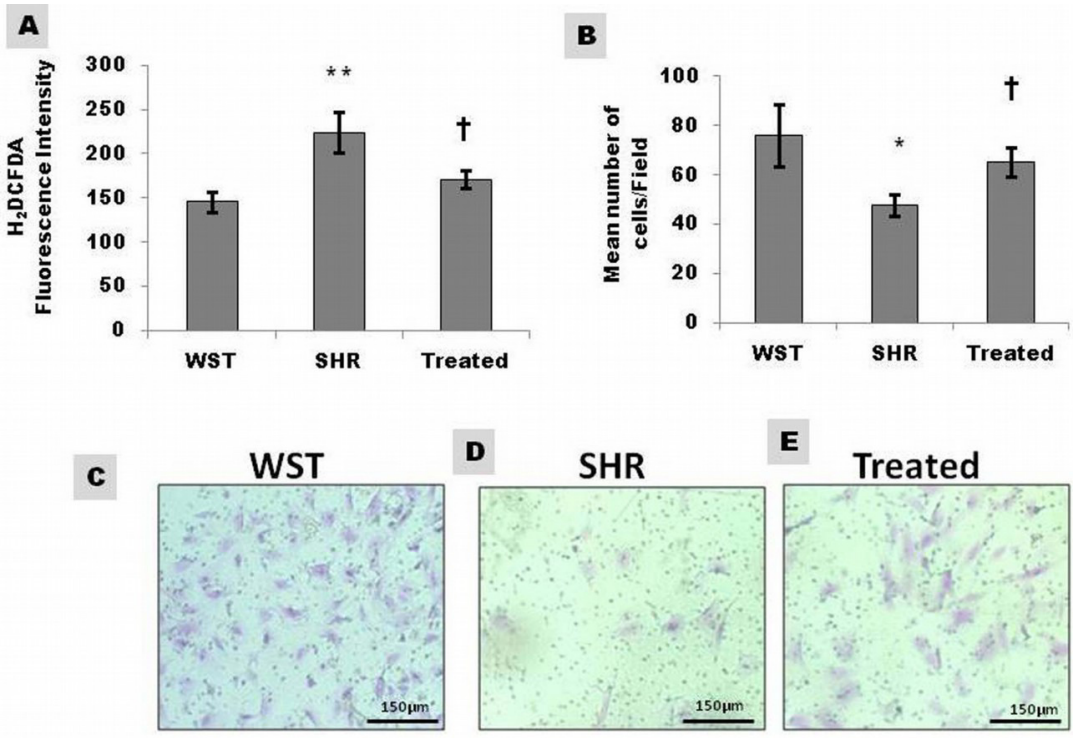

347 Figure 4: Effect of famotidine on intracellular reactive oxygen species (ROS) and migration 348 potential of atrial CSCs

349 A. ROS levels in CSCs represented as $\mathrm{H}_{2}$ DCFDA fluorescence intensity B. Graphical 350 representation and (C-E) Representative images of the migrated cells. Extent of migration is represented as mean number of cells/field. Data presented as mean $\pm \mathrm{SD}$. Variation was analysed by ANOVA followed by Student t-test. (** $p<0.01 \& * \mathrm{p}<0.05$ SHR Vs WST; $\uparrow \mathrm{p}<0.05$ SHR Vs Famotidine) ANOVA $<<0.01$ ( $\mathrm{n}=6 /$ group). 


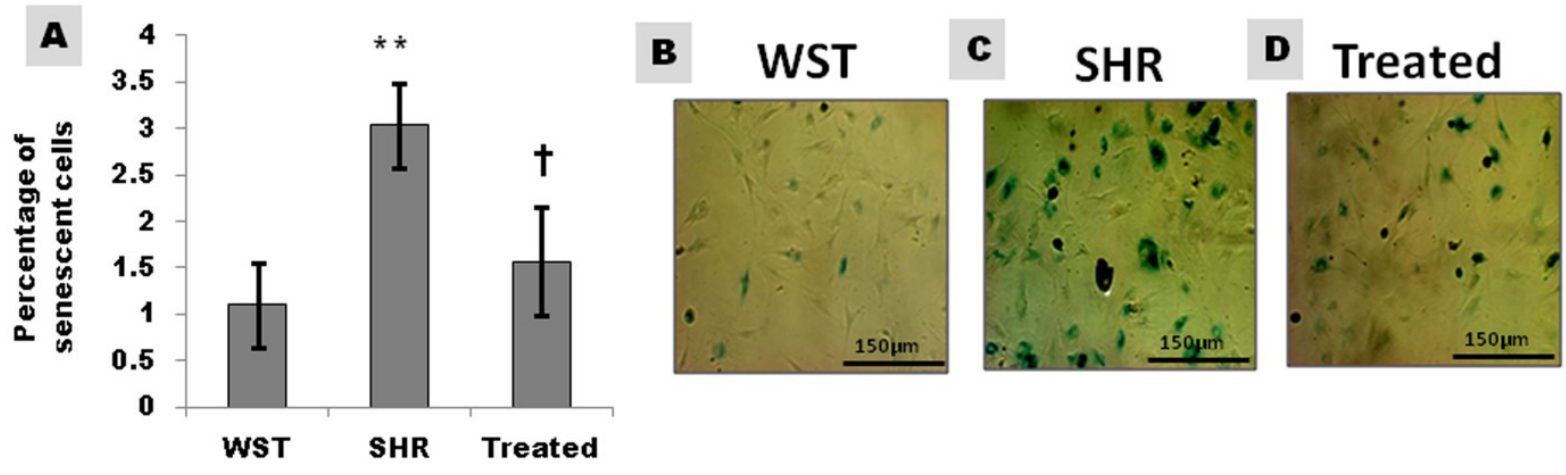

E

$\mathbf{F}$

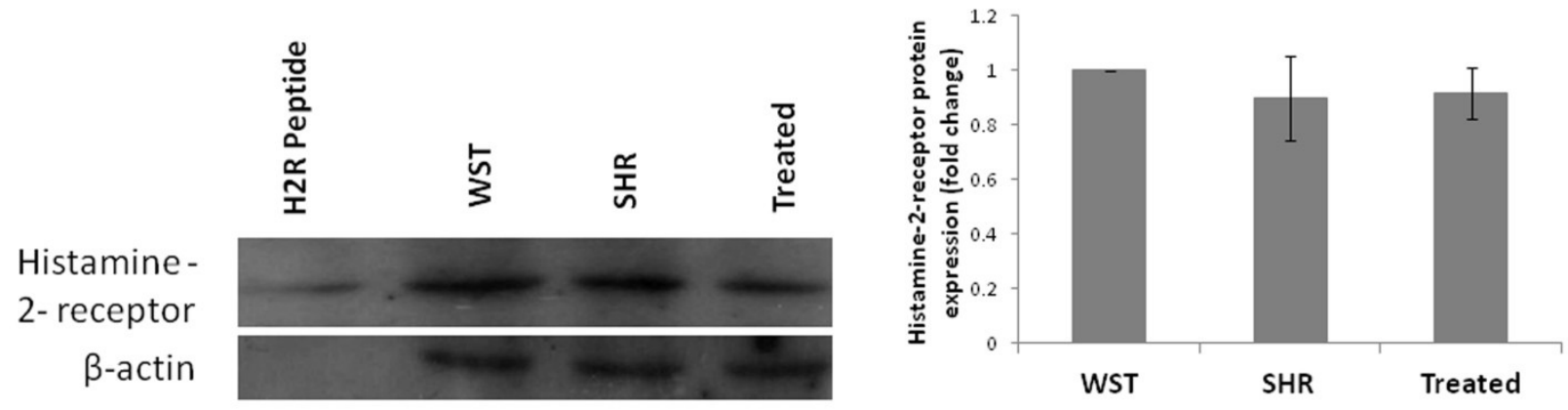

Figure 5: Effect of Famotidine on the senescence and Histamine-2-receptor expression of atrial CSCs.

A.Graphical representation and (B-D) Representative images of senescent CSCs. Proportion of senescent cells is expressed as percentage of the total population. $\mathbf{E} \boldsymbol{\&} \mathbf{F}$. Representative blots and graphical representation of the expression levels of $\mathrm{H} 2 \mathrm{R}$ in atrial CSCs.Data presented as mean \pm SD. Variation was analysed by ANOVA followed by Student t-test. $(* * \mathrm{p}<0.01$ SHR Vs WST; $\uparrow \mathrm{p}<0.05$ SHR Vs Famotidine) ANOVA $\mathrm{p}<0.01$ ( $n=6 /$ group). 